\title{
Suivi des observations de tortues marines sur la côte atlantique française depuis 1988
}

\section{Sea turtles survey on the French Atlantic coast since 1988}

\section{Pierre Morinière \& Florence Dell'Amico}

Aquarium La Rochelle, Centre d'Etudes et de Soins pour les Tortues Marines (C.E.S.T.M.)

tortues@aquarium-larochelle.com

\section{ABSTRACT}

Among the seven species of existing sea turtles, four can be seen in the Bay of Biscay and in the Manche. The leatherback turtle Dermochelys coriacea (Vandelli, 1761) and the loggerhead turtle Caretta caretta (Linnaeus, 1758) are the most seen species whereas the Kemp's ridley turtle Lepidochelys kempii (Garman, 1880) and the green turtle Chelonia mydas (Linnaeus, 1758) are seen more occasionally. The monitoring of the sightings done by the Aquarium La Rochelle and its Center of Studies and Cares for Sea Turtles since 1988 allowed us to identify the main characteristics of the sea turtles present on the studied area.

KEY WORDS: French Atlantic Coast, Dermochelys coriacea, Caretta caretta, Lepidochelys kempii, Chelonia mydas.

\section{RESUMEN}

Entre las siete especies de tortugas marinas que existen, cuatro pueden observarse en el Golfo de Vizcaya y en el Canal de la Mancha La tortuga laúd Dermochelys coriacea (Vandelli, 1761) y la tortuga boba Caretta caretta (Linnaeus, 1758) son las más frecuentes, mientras que la tortuga lora Lepidochelys kempii (Garman, 1880) y la tortuga verde Chelonia mydas (Linnaeus, 1758) se ven ocasionalmente. El seguimiento de avistamientos Ilevado a cabo por el Aquarium de La Rochelle y su centro de estudios y cuidado de tortugas marinas desde 1988 nos ha permitido identificar las características de las tortugas presentes en el área de estudio.

PALABRAS CLAVE: Costa Atlántica Francesa, Dermochelys coriacea, Caretta caretta, Lepidochelys kempii, Chelonia mydas.

\section{LABURPENA}

Existitzen diren zazpi itsas dortoka espezieetatik lau, Bizkaiko Golkoan eta Mantxako kanalean ikus ditzakegu. Larruzko dortoka Dermochelys coriacea (Vandelli, 1761) eta benetazko dortoka Caretta caretta (Linnaeus, 1758) dira ohikoenak, Kemp dortoka Lepidochelys kempii (Garman, 1880) eta berdea Chelonia mydas (Linnaeus, 1758) aldiz oso noizbehinka ikus daitezkeelarik. La Rochelleko akuariumak eta bere baitan duen itsas dortoken zaintza eta ikerketarako zentruak 1988 geroztik itsas dortoken jarraipen bat egin du eta horri eskerrak ikerketa gunean ditugun itsas dortoken ezaugarriak ezagutu ahal izan ditugu.

GAKO-HITZAK: Frantziar kosta atlantiarra, Dermochelys coriacea, Caretta caretta, Lepidochelys kempii, Chelonia mydas.

\section{INTRODUCTION}

Les observations de tortues marines ont été initiés et collectées depuis 1968 sur la côte atlantique française à l'initiative de Raymond Duguy (Dugur, 1968), au départ au nom du Musée d'Histoire naturelle de La Rochelle. Le suivi des échouages est assuré, depuis 1972, en collaboration avec I'Aquarium La Rochelle et son Centre d'Etudes et de Soins pour les Tortues Marines (C.E.S.T.M.). Afin de renforcer les efforts de conservation et de gestion en faveur de ces espèces marines protégées, le suivi satellitaire permet d'étudier les mouvements des tortues marines sur de longues périodes (SENEY, 2010). Le premier suivi par biotélémétrie depuis la côte atlantique française a été réalisé à partir d'une tortue luth femelle subadulte en 1978 (DURON, 1978). Le premier suivi par satellite depuis la côte atlantique française à partir d'une caouanne juvénile a été entrepris en 2008.

\section{MÉTHODE}

Le C.E.S.T.M. recense l'ensemble des échouages et des observations en mer de tortues marines dans le Golfe de Gascogne, dans la mer celtique et en Manche représentant la zone d'étude. II centralise ces informations dans une base de données informatique consacrée à cet inventaire.

Les échouages de tortues marines sont localisés grâce à un réseau d'échouages réparti le long de la zone d'étude. Ce réseau est constitué de bénévoles et d'aquariums publics formés à cette catégorie d'intervention. Les tortues marines vivantes échouées sont recueillies au C.E.S.T.M. qui les soigne et les relâche lorsque leur état le permet. Chaque tortue relâchée est marquée à l'aide d'une bague métallique MNHN (Muséum national d'Histoire naturelle) comportant un numéro unique $\left\langle\mathrm{F}-\mathrm{n}^{\circ} »\right.$ permettant d'identifier l'individu en cas d'observation future ou recapture. Les tortues marines mortes échouées sont autopsiées par le C.E.S.T.M., selon leur état de putréfaction, afin de déterminer la cause de leur mort; des échantillons de tissus sont collectés et conservés à des fins scientifiques.

Des observations en mer de tortues marines sont transmises par des plaisanciers et professionnels de la mer dans le cadre de l'opération «Devenez observateurs des Pertuis», lancée depuis 1996 par I'Aquarium La Rochelle en partenariat avec le Centre de Recherche sur les Mammifères Marins (Université de La Rochelle). Des affi- 


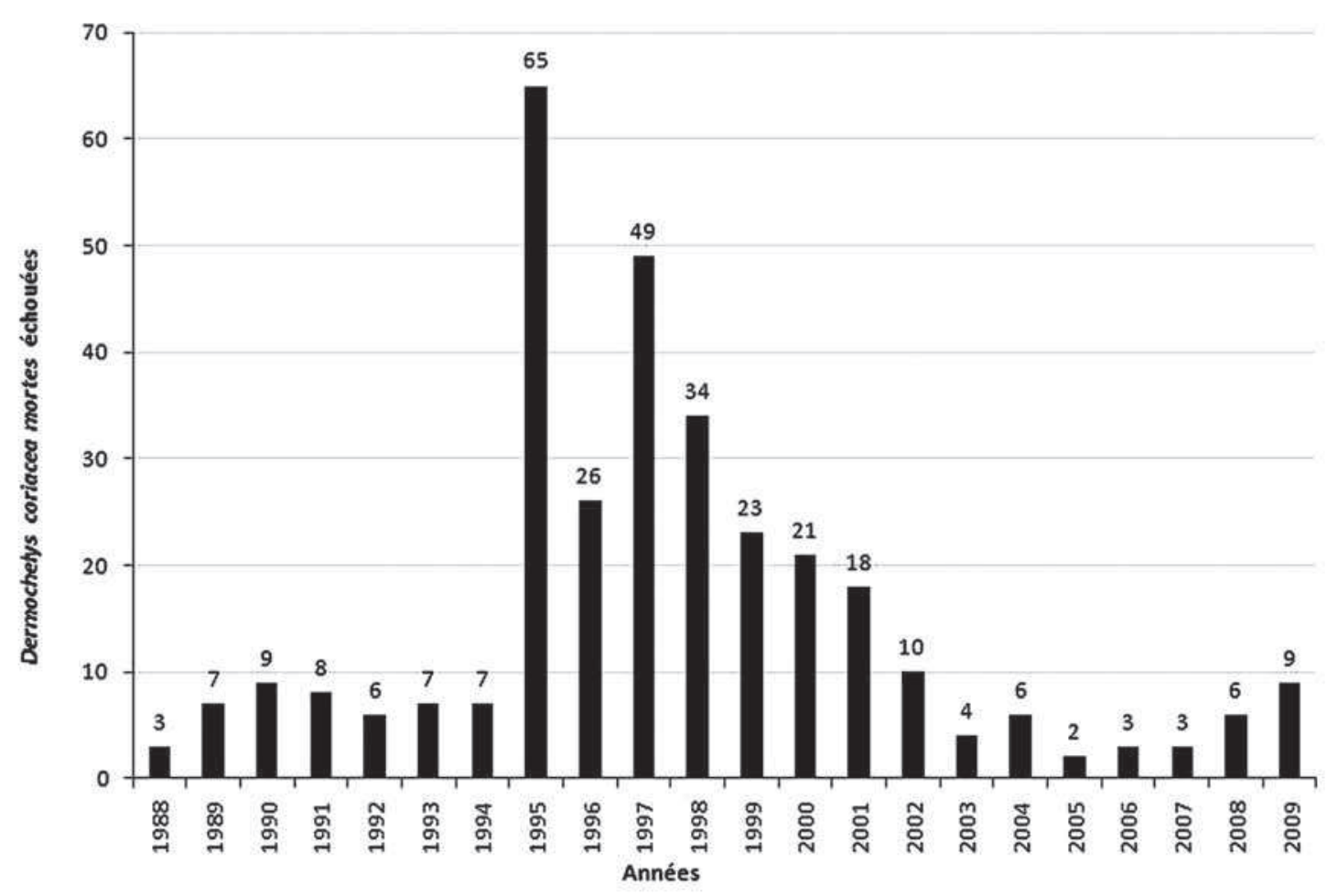

Fig. 1.- D. coriacea mortes échouées au cours des années 1988-2009. / Stranded dead D. coriacea between 1988-2009.

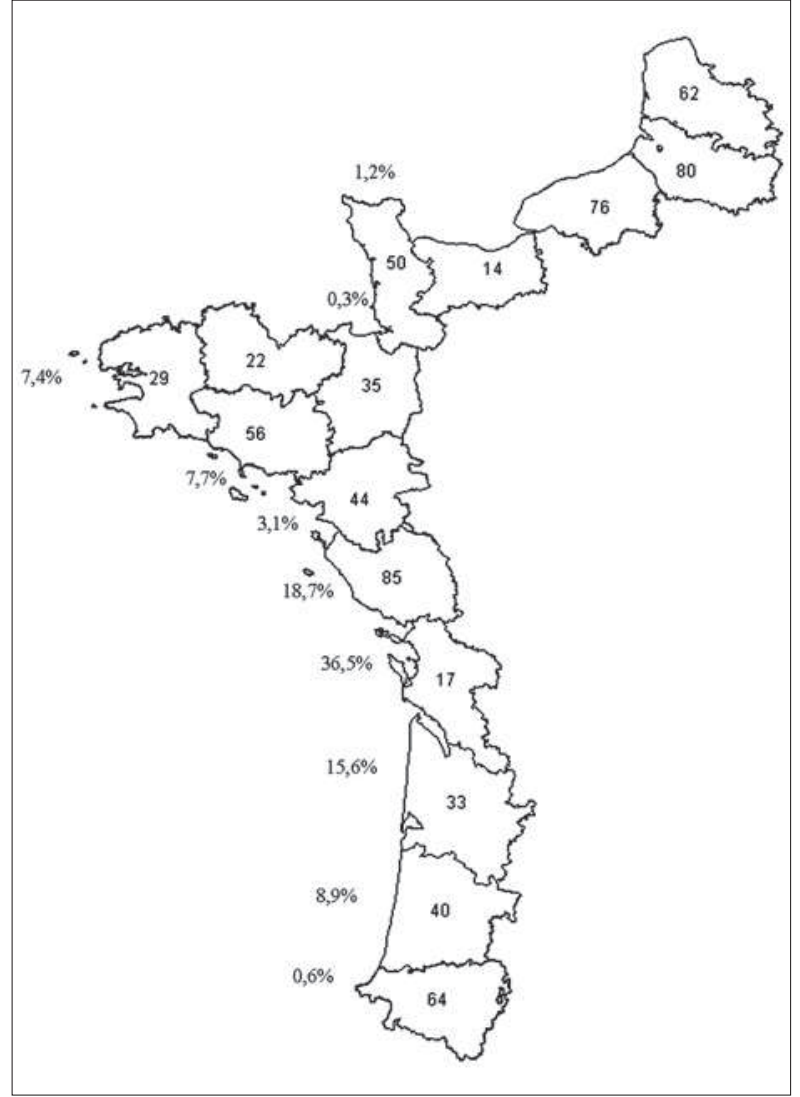

Fig. 2.- D. coriacea échouées observées a la cote Atlantique française. / Observed stranded D. coriacea in the French Atlantic coast departments. ches et des fiches d'observation sont distribuées chaque année aux capitaineries et aux centres nautiques localisés sur la zone d'étude dans cet objectif.

Le premier suivi satellitaire a été réalisé à partir d'une jeune Caretta caretta (Linnaeus, 1758) (SCL: 34,6 cm), échouée vivante dans le sud du Golfe de Gascogne (Lège Cap Ferret (33)), du 29 juillet 2008 au 17 novembre 2008. L'émetteur a été fixé au point le plus haut de la carapace à l'aide de colle epoxy (Power Fasteners ${ }^{\circledR}$ ) selon la méthode décrite par BALAzs et al. (1996). L'individu a été localisé grâce au système satellitaire Argos qui répertorie les positions selon 6 classes de précision (3-1 :<1 km, 0, A et B). Il existe une septième classe $Z$ dont les positions ne sont pas valides. Le trajet de la tortue a été retracé à l'aide des positions transmises dont les classes sont comprises entre 1 et 3.

\section{RÉSULTATS}

Les observations de Dermochelys coriacea(Vandelli, 1761) Distribution annuelle des échouages

Depuis 1988, 326 individus ont été retrouvés morts échoués sur la zone d'étude. Un pic d'échouage de 65 individus a été enregistré en 1995 (Fig. 1).

Les échouages sont constatés toute l'année et sur l'ensemble de la zone d'étude. Toutefois, 69,3\% des échouages sont observés entre les mois de septembre et de décembre et $70,8 \%$ des échouages se répartissent entre les départements de la Gironde (33), de la Charente-Maritime (17) et de la Vendée (85) (Fig. 2). 85,2\% des individus mesurés pré- 
sentaient une classe de taille comprise entre 120 et $169 \mathrm{~cm}$ de longueur courbe de carapace (SCCL). 56,4\% des individus ont été sexés permettant d'identifier 90 mâles et 94 femelles. 83 autopsies ont pu être réalisées dont $42,2 \%$ ont mis en évidence la présence de matières plastique dans le tube digestif. Des indications de prise par des matériels de pêche (filets, orins de casiers à Crustacés, cordages...) ont été retrouvées sur 8,6\% des individus morts échoués.

\section{Distribution annuelle des observations en mer}

Depuis 1988, 1002 observations de tortues luth ont été collectées sur la zone d'étude. Le nombre d'observations a nettement augmenté entre 1996 et 1999 (Fig. 3).

$88,7 \%$ des observations sont réalisées entre les mois de juillet et de septembre. La zone la plus fréquentée par les tortues luth se situe entre les latitudes $45^{\circ} 40 \mathrm{~N}$ et $47^{\circ} \mathrm{N}$ et particulièrement dans le Pertuis breton, zone située entre l'lle de Ré et la côte, depuis les Sables d'Olonne jusqu'à La Rochelle.

Parmi ces observations, 28 individus ont été signalés capturés dans des engins de pêche ou présentant des marques de capture du type filet, orin, ligne ou encore cordage.

\section{Les observations de Caretta caretta (Linnaeus, 1758)}

Distribution et caractéristiques des échouages de tortues caouannes

Au cours de la période étudiée, le nombre d'observations de caouannes sur la zone d'étude est de 292:
232 tortues vivantes et 60 mortes. Ces observations comprennent les tortues mortes échouées, les tortues vivantes échouées et celles trouvées en mer puis ramenées à terre. Deux pics d'observation ont été enregistrés en 1990 (35) et en 2001 (48) (Fig. 4).

Les tortues caouannes sont observées toute l'année. Cependant, 60,6\% des échouages sont réalisés entre les mois de janvier et d'avril. Elles sont principalement rencontrées dans le sud du Golfe de Gascogne et notamment dans les départements de la Gironde (33), des Landes (40) et des Pyrénées Atlantiques (64) qui enregistrent $64,4 \%$ de l'ensemble des observations réalisées (Fig. 5). Sur l'ensemble des tortues mesurées, $66,2 \%$ présentaient une longueur droite de carapace (SCL) comprise entre 15 et $24 \mathrm{~cm}$.

\section{Caractéristiques des symptômes observés chez les tor- tues mises en soin ou autopsiées}

Le C.E.S.T.M. a accueilli 181 C. caretta depuis 1988. Certains symptômes ont été observés chez les tortues mises en soins: la léthargie, I'hypothermie, la flottaison, des infections oculaires, la présence de plaies au niveau de la tête et de la dossière, des sections et des paralysies au niveau des pattes nageoires. 77 autopsies ont été menées sur des individus morts échoués ou décédés en soins. Ces examens ont mis en évidence la présence de bactéries (genres Mycobacterium, Aeromonas, Pseudomonas, Flavobacterium, Aerococcus, Vibrio, Photobacterium, Ateromonas, Myroides et Klebsiella), de champignons (Fusarium

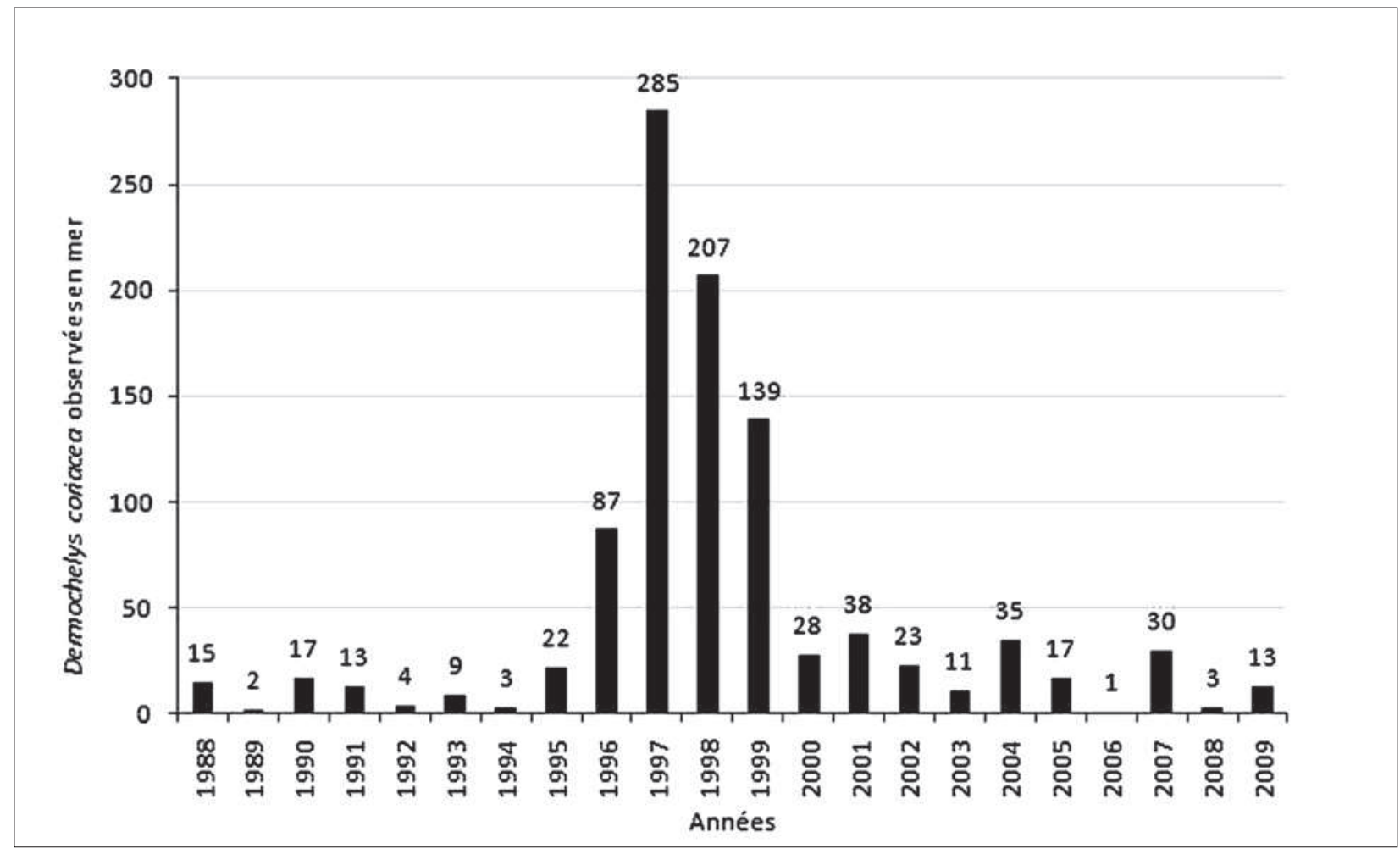

Fig. 3.- D. coriacea observées en mer entre 1988 et 2009. / D. coriacea observed at sea between 1988-2009. 


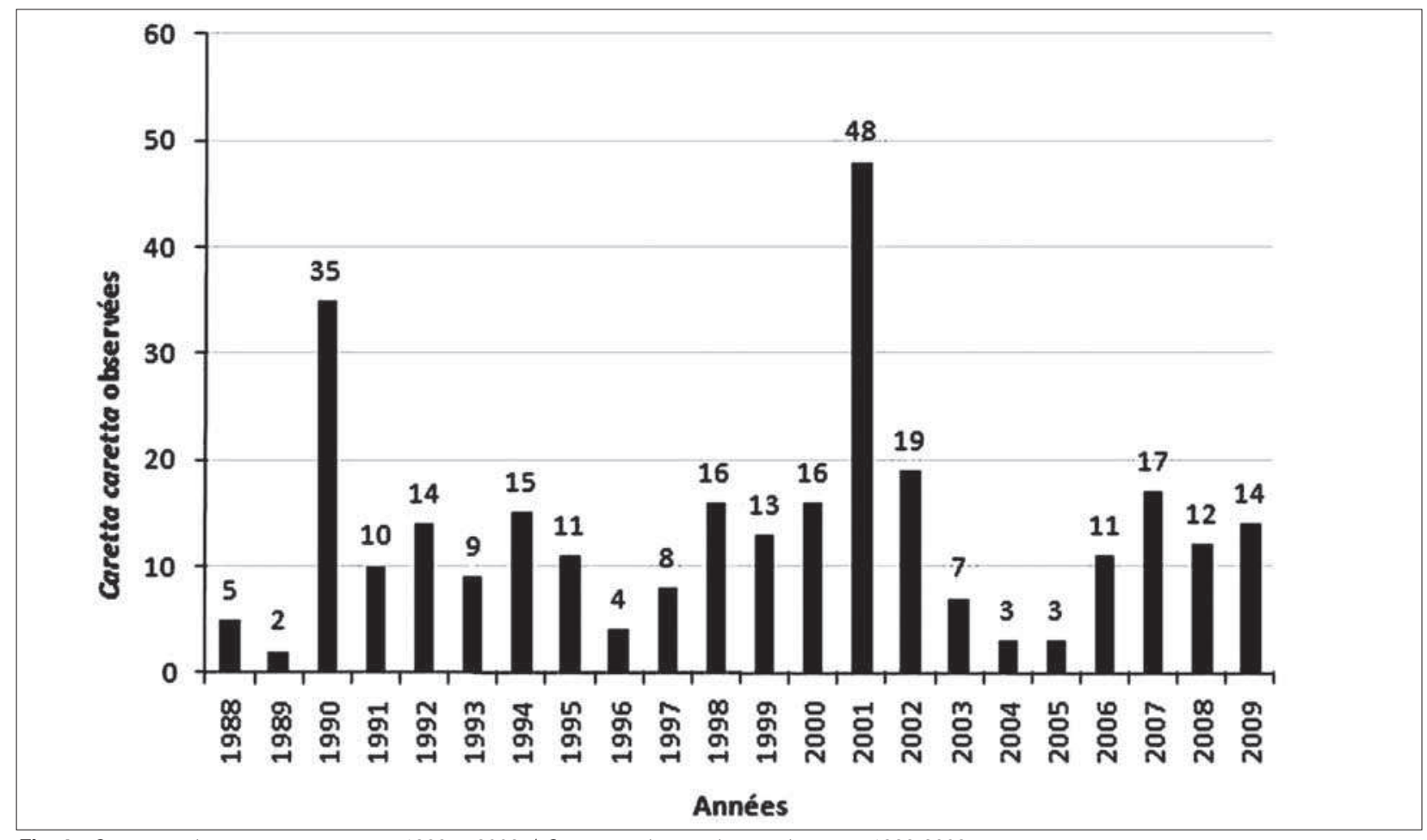

Fig. 4.- C. caretta observées en mer entre 1988 et 2009. / C. caretta observed at sea between 1988-2009.

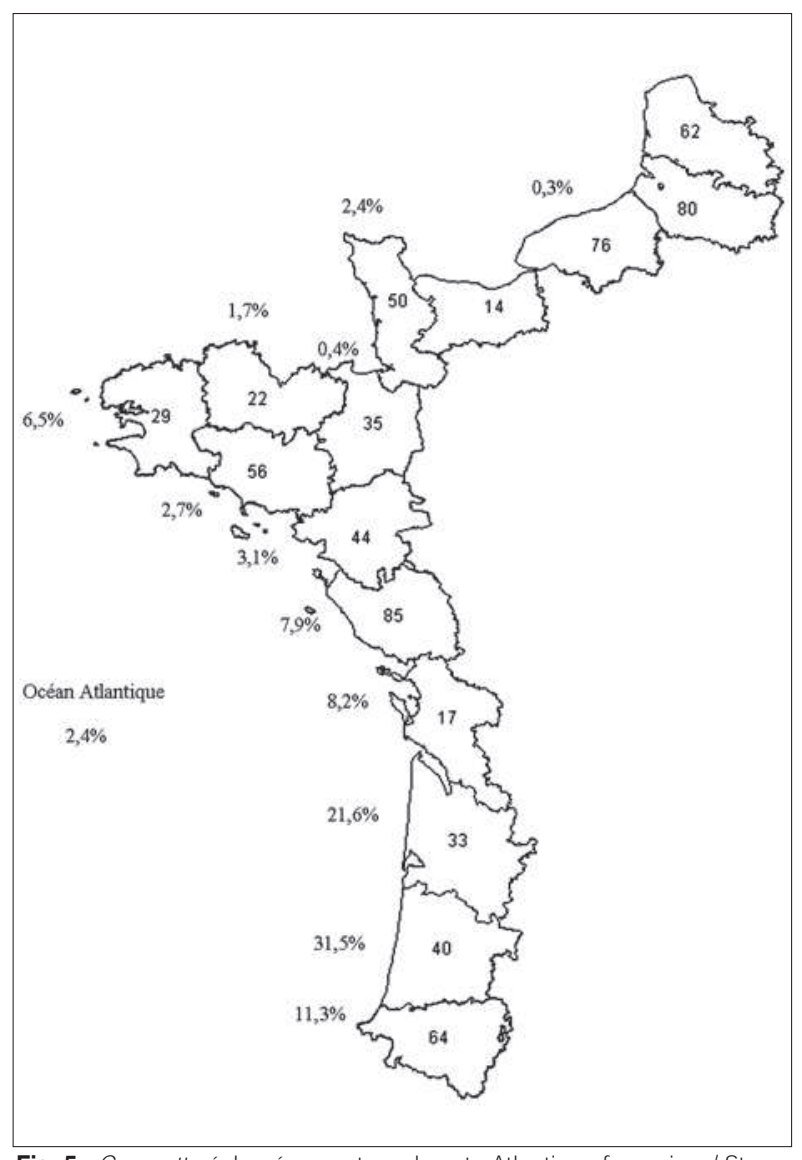

Fig. 5.- C. caretta échouées mortes a la cote Atlantique française. / Stranded dead C. caretta at different French Atlantic coast departments. et Penicillium) et de parasites (Protozoaires). Des matières plastique et des amas de fil de nylon ont été découverts dans l'estomac et l'intestin de 15,6\% des individus.

\section{Remises à l'eau de Caretta caretta}

Depuis 1988, 162 caouannes ont été relâchées en mer. Les relâchers ont été effectués jusqu'en 2008 au large de La Rochelle, à la bouée du Sauerland (4605 N / $1^{\circ} 42$ O). Sur l'ensemble de ces relâchers, 18 tortues ont été à nouveau observées et identifiées grâce à leur numéro de bague MNHN. Ces observations ont été faites quelques jours à quelques mois suite à leur relâcher et au sud du lieu de leur remise à l'eau.

En 2009, les relâchers ont été entrepris depuis la pointe Nord-Est de I'lle de Ré (17) (46¹4 N / $01^{\circ} 32$ O).

\section{Premier suivi satellitaire}

Le premier émetteur satellitaire a été posé sur une jeune C. caretta (baguée F-1934) relâchée le 29 juillet 2008 au niveau de la bouée du Sauerland $\left(46^{\circ} 05 \mathrm{~N} / 01^{\circ} 42 \mathrm{O}\right)$; et suivie jusqu'au 17 novembre $2008\left(45^{\circ} 02 \mathrm{~N} / 01^{\circ} 28 \mathrm{O}\right)$, soit pendant 111 jours. Ce premier suivi a mis en évidence le retour à la côte de l'individu directement après son relâcher ainsi que son déplacement très proche de la côte, enfin une orientation globale vers le nord puis vers le sud (Fig. 6). L'individu a été retrouvé le 19 mars 2009 à Getaria en Espagne où il a été recueilli par I'Aquarium de San Sebastian avant d'être reconduit au C.E.S.T.M. Aucune trace de l'émetteur sur sa carapace n'était alors visible. 


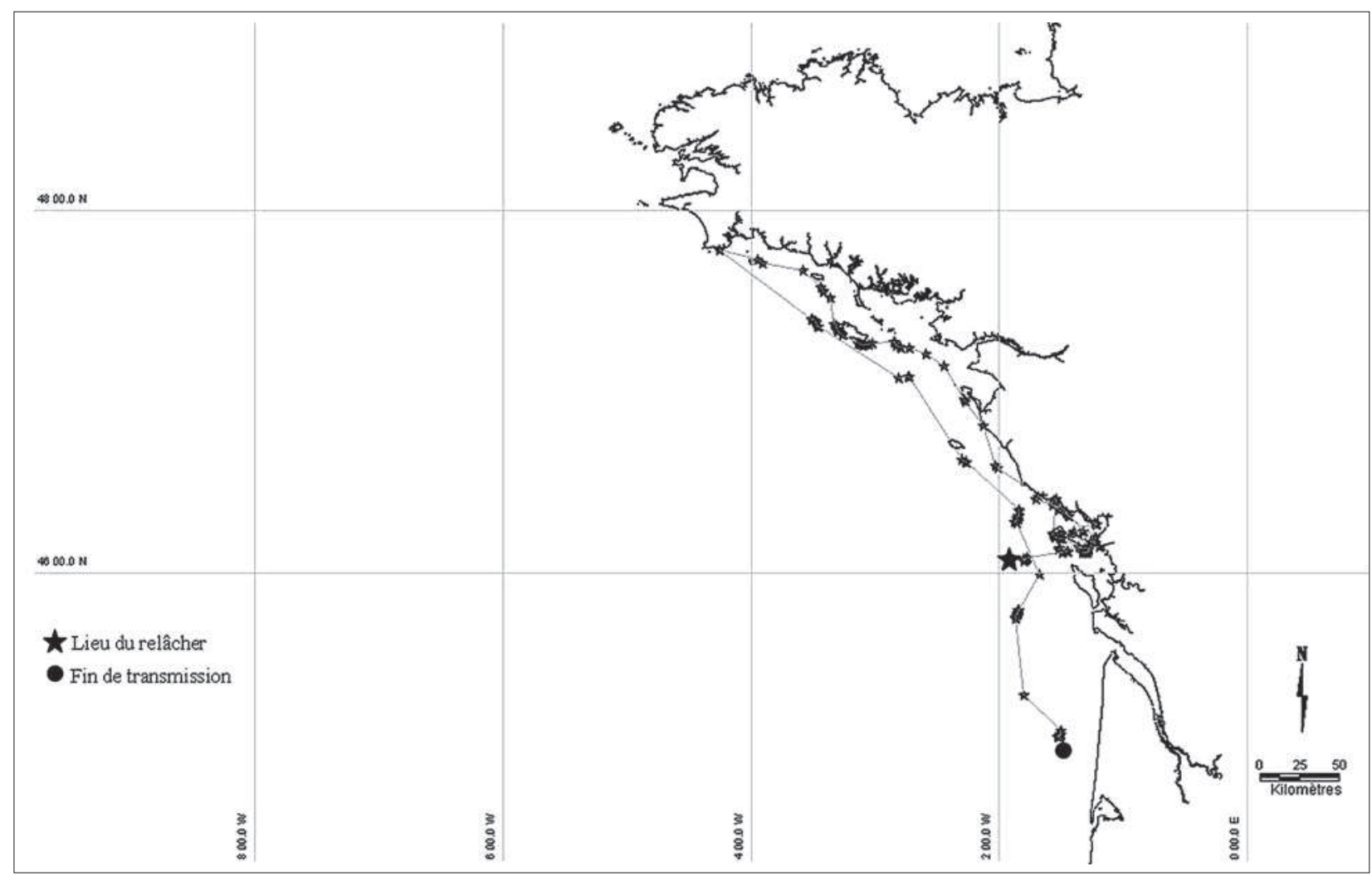

Fig. 6.- Suivi satellitaire de C. caretta a la cote Atlantique française en 2010. / Satelite tracking of C. caretta in Frenchs Atlantic coast in 2010.

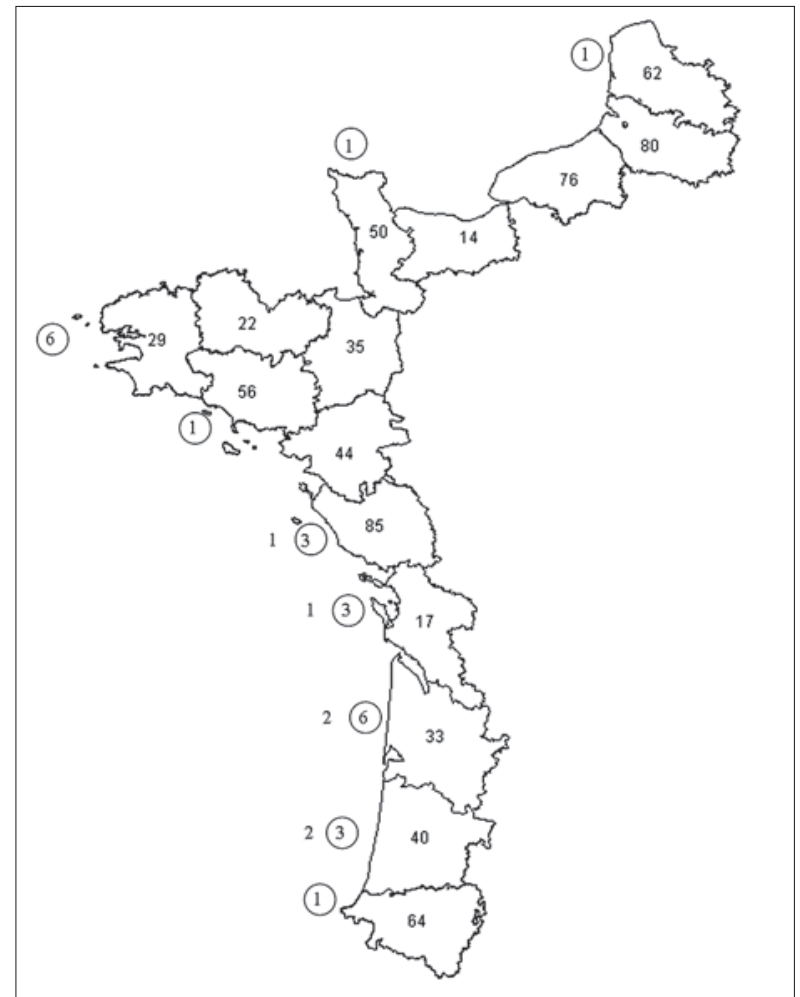

Fig. 7.- L. kempii (cercle) et C. mydas (sans cercle) échouées mortes a la cote Atlantique française entre 1988 et 2010. / Stranded dead L. kempii (circle) and C. mydas (without circle) in French Atlantic coast departments since 1988 to 2010.
Les observations de Lepidochelys kempii (Garman, 1880) et de Chelonia mydas (Linnaeus, 1758)

\section{Lepidochelys kempii}

Depuis 1988, 25 L. kempii ont été retrouvées sur la zone d'étude. Les échouages ont lieu principalement au cours de la période hivernale (68\%) entre les mois de décembre et de février et sur l'ensemble de la zone d'étude. Toutefois, six observations ont été recensées dans le Finistère (29) et six dans la Gironde (33) (Fig. 7). II s'agit d'individus exclusivement juvéniles dont la longueur droite de carapace moyenne $(\mathrm{SCL})$ est de $30 \mathrm{~cm}$.

Treize tortues ont été accueillies au C.E.S.T.M., quatre d'entre elles ont été relâchées. Quinze autopsies ont été pratiquées sur des tortues retrouvées mortes échouées ou décédées en soin et ont mis en évidence la présence de bactéries (Pseudomonas et Mycobacterium), de parasites (Nématodes et Trématodes), de champignons (Fusarium et Aspergillius), de protozoaires (Hexamitia) et de matière plastique dans le tube digestif de deux individus.

\section{Chelonia mydas}

Six C. mydas ont été observées sur la zone d'étude. Les échouages ont été enregistrés en février, en mars et en novembre depuis le département des Landes (40) jusqu'en Vendée (85) (Fig. 7). II s'agit d'individus immatures dont la longueur droite de carapace moyenne (SCL) est de $38 \mathrm{~cm}$. Deux autres individus ont été accueillis au 
C.E.S.T.M. puis relâchés. Quatre autopsies ont été pratiquées et ont révélé la présence de matière plastique dans le gros intestin d'un individu, un œedème pulmonaire et un cas d'infection par Mycobacterium marinarum.

\section{DISCUSSION ET CONCLUSIONS}

Ce suivi a permis d'une part de mettre en évidence la présence de quatre espèces de tortues marines dans le Golfe de Gascogne, en Mer Celtique ainsi qu'en Manche: D. coriacea, C. caretta, L. kempii et C. mydas elle a permis d'autre part de présenter les principales caractéristiques de chacune de ces espèces protégées.

L'espèce $D$. coriacea est observée régulièrement dans la zone d'étude depuis 1988. II s'agit d'individus sub-adultes et adultes (JAmEs et al., 2007) observés en mer l'été en train de s'alimenter de Rhizostomes (DURON, 1978; Dugur et al., 1980) dans la zone des Pertuis charentais et principalement dans la zone du Pertuis breton et quelquefois retrouvés morts échoués en automne et au début de l'hiver, principalement sur les plages bordant les départements de la Charente-Maritime (17), de la Vendée (85) et de la Gironde (33). Dans l'océan Atlantique, les tortues luth entreprennent de longues migrations entre les sites de ponte et les sites d'alimentation (CAUT et al., 2009) et sont observées de façon saisonnière sur la côte est canadienne ainsi qu'en Europe du Nord (DoytE et al., 2007). Des analyses génétiques réalisées sur des échantillons de muscle de tortues luth échouées mortes sur la côte atlantique française sont en cours d'exploitation par la NOAA (Californie) et permettront d'apporter des précisions sur leur origine. Les matières plastique et les interactions avec les engins de pêche sont les deux principaux facteurs de mortalité mis en évidence par les autopsies de ces individus retrouvés morts échoués (Duguy et al., 1998 et 2000).

L'espèce $C$. caretta est également régulièrement observée sur la zone d'étude en particulier dans la partie sud du Golfe de Gascogne qui enregistre plus de la moitié des observations $(64,4 \%)$. Les jeunes individus vivants s'échouent au cours de la période hivernale et au début du printemps et souffrent principalement d'hypothermie. En effet, les symptômes d'hypothermie apparaissent lorsque les eaux descendent en-dessous de $15^{\circ} \mathrm{C}$ et à $10^{\circ} \mathrm{C}$ les caouannes étant alors léthargiques et «flottantes» (MILTON et al., 2003) Leur passage au C.E.S.TM. permet de rétablir leur état physiologique avant de les remettre à l'eau en été au large de La Rochelle. Des analyses réalisées à partir de I'ADN mitochondrial ont permis de valider I'hypothèse que les jeunes caouannes retrouvées dans l'est Atlantique sont originaires des plages de ponte situées dans l'Atlantique de l'ouest (LAURENT et al., 1993 et 1998; Bolten et al., 1998). Des analyses génétiques, en cours de réalisation par la Estación Biologica de Doñana (Sevilla, Espagne) sur des échantillons de muscle, de sang et de peau de C. caretta retrouvées mortes ou vivantes échouées sur la côte atlantique française, permettront de confirmer cette hypothèse.

Le premier suivi par satellite montre que la tortue séjourne dans les eaux littorales de la côte atlantique française pendant plusieurs mois. Le Golfe de Gascogne serait une zone d'habitat et d'alimentation temporaire pour ces jeunes tortues. En 2009, cinq nouveaux suivis satellitaires ont été mis en place. Ils permettront de préciser le temps de résidence de $C$. caretta et les paramètres qui influencent ses trajectoires dans le Golfe de Gascogne.

Les observations de L. kempii sont rares. Quelques individus de tortues de Kemp ont été recensés dans le bassin méditerranéen (TomAs et al., 2003) ainsi qu'en Irlande et au Royaume-Uni (PIERPOINT et al., 2002). La population de la tortue de Kemp est confinée dans le Golfe du Mexique et sur la côte est des Etats-Unis avec un site de ponte principal situé à Rancho Nuevo au Mexique (METz, 2004). Les observations de tortues vertes sont quant à elles exceptionnelles sur la côte atlantique française ainsi qu'au sud du Royaume-Uni et dans la mer du Nord (PIERPoInt et al., 2002). Ces jeunes individus sont transportés depuis les sites de ponte situés dans I'Atlantique Nord-Ouest par le Gulf Stream et la dérive nord Atlantique vers I'Atlantique Nord-Est (ECKERT et al., 2001; Musick et al., 1997).

\section{REMERCIEMENTS}

Les auteurs souhaitent remercier l'ensemble du réseau d'échouage «tortues marines» de la côte atlantique française ainsi que les nombreux observateurs pour leur contribution à ce travail grâce à leurs observations. Nous remercions également le Ministère de l'Ecologie, de l'Energie, du Développement Durable et de la Mer qui soutient les actions menées par l'Aquarium La Rochelle à travers son Centre d'Etudes et de Soins pour les Tortues Marines.

\section{RÉFÉRENCES BIBLIOGRAPHIQUES}

Balazs, G. H., Miya, R. K., Beaver, S. C. 1996. Procedures to attach a satellite transmitter to the carapace of an adult green turtle, Chelonia mydas. In: Proceedings of the Fifteenth Annual Symposium on Sea Turtle Biology and Conservation. J. A. Keinath, D. E. Barnard, J. A. Musick, B. A. Bell, (Compilers): 21-26. NOAA Technical Memorandum NMFS-SWFSC-387. Miami.

Bolten, A. B., Buorndal, K. A., Martins, H. R., Dellinger, T., BiscolTo, M., Encalada, S. E., Bowen, B. W. 1998. Transatlantic developemental migrations of loggerhead sea turtles demonstrated by mtDNA sequence analysis. Ecol. Appl. 8(1): 1-7.

Caut, S., Fossette, S., Guirlet, E, Angulo, E., Das, K., Girondot, M., GEORGES, J. Y. 2009. Isotope analysis reveals foraging area dichotomy for Atlantic leatherback turtles. Plos One 3(3): e1845.

DOyle, T. K., Houghton, D. R., O'SúllLeabHÁin, P. F., Hobson, V. J., Marnell, F., DavenPORT, J., Hays G. C. 2007. Leatherback turtles satellite-tagged in European waters. Endang. Species Res. 3: 15.

Dugur, R. 1968. Note sur la fréquence de la tortue luth Dermochelys coriacea L. près des côtes de la Charente-Maritime. Ann. Soc. Sci. nat. Charente-Maritime 4 (8) : 8-16.

Duguy, R., Duron, M., Alzieu, C. 1980. Observations de tortues luth (Dermochelys coriacea) dans les Pertuis Charentais en 1979. Ann. Soc. Sci. nat. Charente-Maritime 6 : 681-691.

Duguy, R., Moriniėre, P., Le Milinaire, C. 1998. Facteurs de mortalité observés chez les tortues marines dans le golfe de Gascogne. Oceanol. Acta 21 (2): 383 - 388. 
Duguy, R., Moriniėre, P., Spano, M. A. 2000. Ingestion de déchets flottants par la tortue luth Dermochelys coriacea (Vandelli, 1761) dans le golfe de Gascogne. Ann. Soc. Sci. nat. Charente-Maritime 8 (9): 1035-1038.

Duron, M. 1978. Contribution à l'étude de la biologie de Dermochelys coriacea (Linné) dans les Pertuis Charentais. Thèse Univ. Bordeaux, 3ème cycle.

ECkert, K. L., Abreu Grobols, F. A. (Eds). 2001. Proceedings of theregional meeting: "Marine turtle conservation in the wider caribbean region: A dialogue for effective regional management" Santo Domingo, 16-18 November 1999. WIDECAST, IUCNMTSG, WWF and UNEP-CEP.

James, M. C., Sherrill-Mix, S. A., Myers, R. A. 2007. Population characteristics and seasonal migrations of leatherback sea turtles at high latitudes. Mar. Ecol. Prog. Ser. 337: 245-254.

Laurent, L., Casale, P., Bradal, M. M., Godley, B. J., Gerosa, G. Broderick, A. C., SChroth, W., SChierwater, B., LeVy, A. M., FregGl, D., Abd el-Mawla, E. M., Hadoud, D. A., Gomati, H. E., DOMINGO, M. HADJICHRISTOPHOROU, M., KoRNARAKY, L.. DEMIRAYAK, F., GaUtier, C. 1998. Molecular resolution of marine turtle stock composition in fishery bycatch: a case study in the Mediterranean. Mol. Ecol. 7: 1529-1542.

LAurent, L., Lescure, J., ExCOFfIEr, L., BOWEn, B., DOMingo, M., HadJICHRISTOPHOROU, M., KORNARAKY, L., TRABUCHET, G. 1993. Genetic studies of relationship between Mediterranean and Atlantic populations of loggerhead turtle Caretta caretta with mitochondrial marker. C. R. Acad. Sci. Paris 316: 1233-1239
Metz, T. L. 2004. Factors influencing Kemp's ridley sea turtle (Lepidochelys kempii) distribution in nearshore waters and implications for management. Ph. D. Thesis. Texas A\&M University.

MiLton, S. L., LUTZ, P. L. 2003. Physiological and genetic responses to environmental stress. In: The biology of sea turtles vol II. P. L. Lutz, J. A. Musick, J. Wyneken (Eds): 163-197. CRC Press. Florida.

Musıck, J. A., Lımpus, C. J. 1997. Habitat utilization and migration in juvenile sea turtles. In: The Biology of Sea Turtles. P. L. Lutz, J. A. Musick (Eds): 137-163. CRC Press. Florida

Pierpoint, C., Penrose, R. 2002. «Turtle» A database of marine turtle records for the UK \& Eire Marine Environmental Monitoring.

Seney, E. E., Higgins, B. M., LAndry, A. M. JR. 2010. Satellite transmitter attachment techniques for small juvenile sea turtles. J. Exp. Mar. Biol. Ecol. 384: 61-67.

Tómas, J., Formia, A., Fernandez, M., Raga, J. A. 2003. Occurrence and genetic analysis of a Kemp's Ridley sea turtle (Lepidochelys kempii) in the Mediterranean Sea. Sci. Mar. 67 (3): 367-369. 